\title{
On Ellipsoids Attached to Root Systems
}

\author{
Anatoli Loutsiouk \\ Department of Civil Engineering, King Mongkut University of Technology Thonburi, Bangkok, Thailand \\ Email: loutsiouk.ana@kmutt.ac.th
}

Received 25 February 2016; accepted 8 August 2016; published 15 August 2016

\begin{abstract}
For any finite-dimensional complex semisimple Lie algebra, two ellipsoids (primary and secondary) are considered. The equations of these ellipsoids are Diophantine equations, and the Weyl group acts on the sets of all their Diophantine solutions. This provides two realizations (primary and secondary) of the Weyl group on the sets of Diophantine solutions of the equations of the ellipsoids. The primary realization of the Weyl group suggests an order on the Weyl group, which is stronger than the Chevalley-Bruhat ordering of the Weyl group, and which provides an algorithm for the Chevalley-Bruhat ordering. The secondary realization of the Weyl group provides an algorithm for constructing all reduced expressions for any of its elements, and thus provides another way for the Chevalley-Bruhat ordering of the Weyl group.
\end{abstract}

\section{Keywords}

Complex Semisimple Lie Algebra, Cartan Subalgebra, Weyl Group, Cartan Matrix, Primary and Secondary Ellipsoids, Diophantine Equations, Geometric Realizations, Coxeter Relations, Dynkin Diagram, Chevalley-Bruhat Ordering, Reduced Expressions

\section{Introduction}

For any complex semisimple Lie algebra, there are a number of mathematical objects that are traditionally attached to it, and which determine it to some extent. The most widely used mathematical objects are: the Dynkin diagram, the Cartan matrix, the system of positive roots, the system of simple roots, the Weyl group, the universal enveloping algebra, etc. These objects have proved their usefulness in dealing with complex semisimple Lie algebras, and most of them have been generalized in order to deal with the new classes of mathematical structures, such as Kac-Moody algebras, superalgebras, quantum groups and Coxeter systems.

In this paper, two alternative mathematical objects are defined for any complex semisimple Lie algebra $\mathfrak{G}$. These objects are ellipsoids in the real linear space $\mathbb{R}^{n}$, where $n$ is the rank of $\mathfrak{G}$.

Given a complex semisimple Lie algebra $\mathfrak{G}$ and a Cartan subalgebra $\mathfrak{H}$, the pair $(\mathfrak{G}, \mathfrak{H})$ determines the system of roots $\Delta$, a subsystem $\Delta^{+}$of all positive roots, and the subsystem $\Pi$ of all simple roots, see [1] [2]. In the space $\mathbb{R}^{n}$, define an inner product $<,>$ in such a way that

$$
\frac{2<e_{i}, e_{j}>}{<e_{i}, e_{i}>}=a_{i j} \text {, }
$$


where $a_{i j}$ is an element of the Cartan matrix $A$ defined by the system $\Pi$ of simple roots, and $e_{i}$ is an element of the standard basis of $\mathbb{R}^{n}$, which we identify with the simple root $\alpha_{i}$.

Denote by $\mathbb{R}_{+}^{n}$ the subset of $\mathbb{R}^{n}$ consisting of all $x=\left(x_{1}, x_{2}, \ldots, x_{n}\right)$ with $x_{i} \geq 0$ for $i=1,2, \ldots, n$. Then, $\Delta^{+}$can be considered as a subset of $\mathbb{R}_{+}^{n}$. Let $\delta$ be the half-sum of all positive roots in this realization of $\Delta^{+}$. The element $\delta \in \mathbb{R}_{+}^{n}$ satisfies the equation

$$
A \delta=\tilde{1}=(1, \ldots, 1),
$$

that will be used in this paper.

We assume the linear space $\mathbb{R}^{n}$ to be partially ordered as follows: $x \leq y$ if and only if for any $1 \leq i \leq n$ we have $x_{i} \leq y_{i}$.

\section{Primary and Secondary Ellipsoids}

The principal object of study in this paper is the subset of $\mathbb{R}^{n}$ defined by the equation

$$
<x, x-2 \delta>=0 \text {. }
$$

This equation determines an ellipsoid in the space $\mathbb{R}^{n}$ with the center at the point $\delta$, and with the extreme

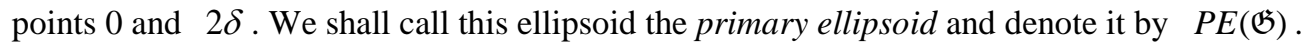

For the case of $\mathfrak{G}$ belonging to the class $A_{1}$, Equation (3) acquires the form

$$
x^{2}-x=0,
$$

and so the primary ellipsoid in this case is the two-point subset $\{0,1\}$ of $\mathbb{R}^{1}$.

For $\mathfrak{G}$ belonging to the class $A_{1} \oplus A_{1}$, Equation (3) becomes

$$
\left(x_{1}^{2}-x_{1}\right)+\left(x_{2}^{2}-x_{2}\right)=0,
$$

which is the equation of a circle passing through the points $(0,0),(0,1),(1,0)$, and $(1,1)$ of $\mathbb{R}^{2}$.

In cases of $A_{2}, B_{2}$, and $G_{2}$, Equation (3) turns to be

$$
\left(x_{1}^{2}-x_{1}\right)+k\left(x_{2}^{2}-x_{2}\right)-k x_{1} x_{2}=0 \text {, }
$$

with $\mathrm{k}=1$ for $A_{2}, \mathrm{k}=2$ for $B_{2}$, and $\mathrm{k}=3$ for $G_{2}$, which in all the three cases is equation of an ellipse passing through the points $(0,0),(0,1),(1,0),(1,2)$, and $2 \delta$.

In general case, the easiest way to write down Equation (3) in coordinate form is through the Dynkin diagram for the semisimple Lie algebra $\mathfrak{G}$, [2] [3]. The Dynkin diagram has $n$ vertices $a_{i}$. Each vertice has a weight denoted by $k_{i}$, which is an integer equal to 1,2 , or 3 . Some of the vertices are connected by links, the number of edges in a link can also be equal to 1,2 , or 3 . For any link connecting verices $a_{i}$ and $a_{j}$, let $l_{i j}=\max \left(k_{i}, k_{j}\right)$, otherwise put $l_{i j}=0$.

Theorem 2.1. Equation (3) in coordinate form is as follows:

$$
\sum_{i=1}^{n} k_{i}\left(x_{i}^{2}-x_{i}\right)-\sum_{1 \leq i<j \leq n} l_{i j} x_{i} x_{j}=0,
$$

where the first sum is taken over all the vertices, and the second sum is taken over all the links in the Dynkin diagram for the complex semisimple Lie algebra $\mathfrak{G}$.

Proof. By direct substitution of the vector $x=\left(x_{1}, \ldots, x_{n}\right)=x_{1} e_{1}+\ldots+x_{n} e_{n}$ into Equation (3).

Remark 2.1. As a matter of fact, the coefficients $l_{i j}$ are not always equal to the number of edges in the link connecting the vertices $a_{i}$ and $a_{j}$, this is essential for the cases $B_{n}$ and $F_{4}$; in all the other cases the coefficients $l_{i j}$ are equal to the number of edges in the link connecting the corresponding vertices.

Owing to the fact that the Cartan matrix is positive definite, Equation (7) is equation of an ellipsoid in the space $\mathbb{R}^{n}$. This ellipsoid contains the origin $0=(0, \ldots, 0)$ and all points $e_{k}=(0, \ldots, 1, \ldots, 0)$, that we identify with the simple roots $\alpha_{k}$. It also contains the points $2 \delta$ and $2 \delta-e_{k}$ for $k=1, \ldots, n$.

For any root $\alpha$, let

$$
m_{\alpha}=\frac{2<\alpha, \delta>}{<\alpha, \alpha>} .
$$

Proposition 2.1. $m_{\alpha}$ is an integer, which is positive if and only if the root $\alpha$ is positive, and it is equal to 
1 if and only if the positive root $\alpha$ is simple.

Proof. Case by case verification.

We shall call the number $m_{\alpha}$ the grade of the root $\alpha$.

Proposition 2.2. For any root $\alpha=\left(a_{1}, a_{2}, \ldots, a_{n}\right) \in \mathbb{R}^{n}$, the element $m_{\alpha} \alpha=\left(m_{\alpha} a_{1}, m_{\alpha} a_{2}, \ldots, m_{\alpha} a_{n}\right)$ belongs to the primary ellipsoid $E(\mathfrak{G})$ defined by Equations (3) or (7).

Proof. It is sufficient to show that the element $m_{\alpha} \alpha$ satisfies Equation (3).

$$
\begin{aligned}
& <m_{\alpha} \alpha, m_{\alpha} \alpha-2 \delta>=m_{\alpha}^{2}<\alpha, \alpha>-m_{\alpha} 2<\alpha, \delta> \\
& =m_{\alpha}<\alpha, \alpha>\left(m_{\alpha}-\frac{2<\alpha, \delta>}{<\alpha, \alpha>}\right)=m_{\alpha}<\alpha, \alpha>\left(m_{\alpha}-m_{\alpha}\right)=0 .
\end{aligned}
$$

We now define one more ellipsoid related to the semisimple Lie algebra $\mathfrak{G}$, and denoted $\operatorname{SE}(\mathfrak{G})$, that we shall call the secondary ellipsoid for $\mathfrak{G}$. For any $x \in P E(\mathfrak{G})$, with $x=\left(x_{1}, \ldots, x_{n}\right)$, and for any $i \in\{1, \ldots, n\}$, we define $h_{i}^{x} \in \mathbb{R} \backslash\{0\}$, if it exists, otherwise we set $h_{i}^{x}=0$, so that

$$
T_{i}(x)=\left(x_{1}, \ldots, x_{i-1}, x_{i}+h_{i}^{x}, x_{i+1}, \ldots, x_{n}\right) \in P E(\mathfrak{G}) .
$$

Such $h_{i}^{x}$ is a unique real number. Consider the vector $h^{x}=\left(h_{1}^{x}, h_{2}^{x}, \ldots, h_{n}^{x}\right) \in \mathbb{R}^{n}$.

\section{Proposition 2.3.}

$$
h^{x}=\tilde{1}-A x=A(\delta-x) .
$$

Proof. By direct substitution of $T_{i}(x)$ with $h_{i}^{x}$ evaluated by formula 10 into Equation (7) of the primary ellipsoid $P E(\mathfrak{G})$.

Observe that if $x$ is an integral vector, that is a vector with all integer components, then $h^{x}$ is an integral vector as well. The set $S E(\mathfrak{G})$ is the set of all such vectors $h^{x} \in \mathbb{R}^{n}$ as $x$ runs through the primary ellipsoid $P E(\mathfrak{G})$.

Theorem 2.2. The subset $S E(\mathfrak{G})$ of $\mathbb{R}^{n}$ is an ellipsoid, which is described by the equation

$$
<A^{-1}(\tilde{1}-h), A^{-1}(\tilde{1}+h)>=0,
$$

or, equivalently,

$$
<A^{-1}(h), A^{-1}(h)>=<\delta, \delta>.
$$

Proof. By direct calculation.

In coordinate form, for the case $A_{1}$, the equation of secondary ellipsoid has the form:

$$
h^{2}=1 .
$$

So, in this case, the secondary ellipsoid is the two-point subset $\{1,-1\}$ of the real line.

For $\mathfrak{G}$ belonging to the class $A_{1} \oplus A_{1}$, the equation of secondary ellipsoid becomes

$$
h_{1}^{2}+h_{2}^{2}=2 \text {, }
$$

which is the equation of the circle centered at the origin and passing through the points $(1,1)$, and $(-1,-1)$.

In cases of $A_{2}, B_{2}$, and $G_{2}$, the equation of secondary ellipsoid in coordinate form turns to be

$$
h_{1}^{2}+k h_{2}^{2}+k h_{1} h_{2}=2 k+1,
$$

with $k=1$ for $A_{2}, \mathrm{k}=2$ for $B_{2}$, and $k=3$ for $G_{2}$, respectively, which in all the three cases is the equation of an ellipse passing through the points $(1,1)$, and $(-1,-1)$, and with the center at the origin.

The primary ellipsoid is determined by the secondary ellipsoid in accordance with the formula

$$
x_{h}=A^{-1}(\tilde{1}-h) .
$$

Theorem 2.3. The equations of secondary ellipsoids in coordinate form for the simple Lie algebras of the four infinite series $A_{n}, B_{n}, C_{n}$, and $D_{n}$ are as follows:

Case $A_{n}$ :

$$
\sum_{j=1}^{n} j(n+1-j)\left(h_{j}^{2}-1\right)+2 \sum_{1 \leq j<k \leq n} j(n+1-k)\left(h_{j} h_{k}-1\right)=0 .
$$


Case $B_{n}$ :

$$
4 \sum_{j=1}^{n-1} j\left(h_{j}^{2}+h_{j} h_{n}-2\right)+n\left(h_{n}^{2}-1\right)+8 \sum_{j=1}^{n-2} j \sum_{k=j+1}^{n-1}\left(h_{j} h_{k}-1\right)=0 .
$$

Case $C_{n}$ :

$$
\sum_{j=1}^{n} j\left(h_{j}^{2}-1\right)+2 \sum_{1 \leq j<k \leq n} j\left(h_{j} h_{k}-1\right)=0 .
$$

Case $D_{n}$ :

$$
\begin{aligned}
& 4 \sum_{j=1}^{n-2} j\left(h_{j}^{2}+h_{j} h_{n-1}+h_{j} h_{n}-3\right)+n\left(h_{n-1}^{2}+h_{n}^{2}-2\right) \\
& +8 \sum_{j<k<n-1} j\left(h_{j} h_{k}-1\right)+2(n-2)\left(h_{n-1} h_{n}-1\right)=0 .
\end{aligned}
$$

The equations of secondary ellipsoids for the remaining 5 exceptional cases of simple Lie algebras are given next as follows:

Case $E_{6}$ :

$$
\begin{aligned}
& 2\left(h_{1}^{2}-1\right)+3\left(h_{2}^{2}-1\right)+5\left(h_{3}^{2}-1\right)+9\left(h_{4}^{2}-1\right)+5\left(h_{5}^{2}-1\right)+2\left(h_{6}^{2}-1\right) \\
& +3\left(h_{1} h_{2}-1\right)+5\left(h_{1} h_{3}-1\right)+6\left(h_{1} h_{4}-1\right)+4\left(h_{1} h_{5}-1\right)+2\left(h_{1} h_{6}-1\right) \\
& +6\left(h_{2} h_{3}-1\right)+9\left(h_{2} h_{4}-1\right)+6\left(h_{2} h_{5}-1\right)+3\left(h_{2} h_{6}-1\right)+12\left(h_{3} h_{4}-1\right) \\
& +8\left(h_{3} h_{5}-1\right)+4\left(h_{3} h_{6}-1\right)+12\left(h_{4} h_{5}-1\right)+6\left(h_{4} h_{6}-1\right)+5\left(h_{5} h_{6}-1\right)=0 .
\end{aligned}
$$

Case $E_{7}$ :

$$
\begin{aligned}
& 4\left(h_{1}^{2}-1\right)+7\left(h_{2}^{2}-1\right)+12\left(h_{3}^{2}-1\right)+24\left(h_{4}^{2}-1\right)+15\left(h_{5}^{2}-1\right)+8\left(h_{6}^{2}-1\right) \\
& +3\left(h_{7}^{2}-1\right)+8\left(h_{1} h_{2}-1\right)+12\left(h_{1} h_{3}-1\right)+16\left(h_{1} h_{4}-1\right)+12\left(h_{1} h_{5}-1\right) \\
& +8\left(h_{1} h_{6}-1\right)+4\left(h_{1} h_{7}-1\right)+16\left(h_{2} h_{3}-1\right)+24\left(h_{2} h_{4}-1\right)+18\left(h_{2} h_{5}-1\right) \\
& +12\left(h_{2} h_{6}-1\right)+6\left(h_{2} h_{7}-1\right)+32\left(h_{3} h_{4}-1\right)+24\left(h_{3} h_{5}-1\right)+16\left(h_{3} h_{6}-1\right) \\
& +8\left(h_{3} h_{7}-1\right)+36\left(h_{4} h_{5}-1\right)+24\left(h_{4} h_{6}-1\right)+12\left(h_{4} h_{7}-1\right)+20\left(h_{5} h_{6}-1\right) \\
& +10\left(h_{5} h_{7}-1\right)+8\left(h_{6} h_{7}-1\right)=0 .
\end{aligned}
$$

Case $E_{8}$ :

$$
\begin{aligned}
& 4\left(h_{1}^{2}-1\right)+8\left(h_{2}^{2}-1\right)+14\left(h_{3}^{2}-1\right)+30\left(h_{4}^{2}-1\right)+20\left(h_{5}^{2}-1\right)+12\left(h_{6}^{2}-1\right) \\
& +6\left(h_{7}^{2}-1\right)+2\left(h_{8}^{2}-1\right)+10\left(h_{1} h_{2}-1\right)+14\left(h_{1} h_{3}-1\right)+20\left(h_{1} h_{4}-1\right) \\
& +16\left(h_{1} h_{5}-1\right)+12\left(h_{1} h_{6}-1\right)+8\left(h_{1} h_{7}-1\right)+4\left(h_{1} h_{8}-1\right)+20\left(h_{2} h_{3}-1\right) \\
& +30\left(h_{2} h_{4}-1\right)+24\left(h_{2} h_{5}-1\right)+18\left(h_{2} h_{6}-1\right)+12\left(h_{2} h_{7}-1\right)+6\left(h_{2} h_{8}-1\right) \\
& +40\left(h_{3} h_{4}-1\right)+32\left(h_{3} h_{5}-1\right)+24\left(h_{3} h_{6}-1\right)+16\left(h_{3} h_{7}-1\right)+8\left(h_{3} h_{8}-1\right) \\
& +48\left(h_{4} h_{5}-1\right)+36\left(h_{4} h_{6}-1\right)+24\left(h_{4} h_{7}-1\right)+12\left(h_{4} h_{8}-1\right)+30\left(h_{5} h_{6}-1\right) \\
& +20\left(h_{5} h_{7}-1\right)+10\left(h_{5} h_{8}-1\right)+16\left(h_{6} h_{7}-1\right)+8\left(h_{6} h_{8}-1\right)+6\left(h_{7} h_{8}-1\right)=0 .
\end{aligned}
$$

Case $F_{4}$ :

$$
\begin{aligned}
& \left(h_{1}^{2}-1\right)+3\left(h_{2}^{2}-1\right)+6\left(h_{3}^{2}-1\right)+2\left(h_{4}^{2}-1\right)+3\left(h_{1} h_{2}-1\right) \\
& +4\left(h_{1} h_{3}-1\right)+2\left(h_{1} h_{4}-1\right)+8\left(h_{2} h_{3}-1\right)+4\left(h_{2} h_{4}-1\right)+6\left(h_{3} h_{4}-1\right)=0 .
\end{aligned}
$$

Case $G_{2}$ :

$$
\left(h_{1}^{2}-1\right)+3\left(h_{2}^{2}-1\right)+3\left(h_{1} h_{2}-1\right)=0 .
$$

Proof. By direct calculation.

Corollary 2.1. A case-by-case examination of the equations of secondary ellipsoids in coordinate form (17)- 
(25) has shown that these equations can be written in a unified form as follows:

$$
\sum_{i=1}^{n} k_{i} b_{i i}\left(h_{i}^{2}-1\right)+2 \sum_{1 \leq i<j \leq n} k_{i} b_{i j}\left(h_{i} h_{j}-1\right)=0,
$$

where $k_{i}$ is the weight of the $i$-th vertice in the Dynkin diagram, and $b_{i i}, b_{i j}$ are elements of the matrix $A^{-1}$ (the inverse matrix for the Cartan matrix $A$ ).

It is clear that this equation is valid for any complex semisimple Lie algebra, and not just for the simple Lie algebras. By multiplying Equation (26) with $\operatorname{det} A$, we get an equation with all coefficients being nonnegative integers.

\section{Diophantine Equations Derived from the Equations of Ellipsoids}

Consider Equation (7) of primary ellipsoid and Equation (26) of secondary ellipsoid as Diophantine equations. This means that we are now concerned with only those solutions to these equations, which have all their components integers. We shall now explore the sets of all solutions of these Diophantine equations. These sets are nonempty and finite. We shall denote them by $P D(\mathfrak{G})$ and $S D(\mathfrak{G})$ respectively. The set $P D(\mathfrak{G})$ contains the elements $0=(0, \cdots, 0)$ and $2 \delta$, and all the standard basis elements $e_{i}=(0,, 1, ., 0)$, and also $2 \delta-e_{i}$, and even more, as follows from Proposition 2.2, for any positive root $\alpha$, the element $m_{\alpha} \alpha$, with $m_{\alpha}$ being the grade of the positive root $\alpha$, also belongs to the set $P D(\mathfrak{G})$. Formula 10 implies that for any $x \in P D(\mathfrak{G})$ the vector $h^{x}=\tilde{1}-A x$ belongs to $S D(\mathfrak{G})$, and this assignment is injective.

Although the extreme points of the primary ellipsoid $P E(\mathfrak{G})\left(0\right.$ and $2 \delta$ ) both belong to $\mathbb{R}_{+}^{n}$, the primary ellipsoid is not competely in $\mathbb{R}_{+}^{n}$; there exists an open neighbourhood $U$ of the origin (in the primary ellipsoid) all of whose elements, except the origin itself, have at least one strictly negative component. This conclusion follows from the form of Equation (7). Formula 9 implies that for any $x \in P D(\mathfrak{G})$ and for any positive integer $i$ with $1 \leq i \leq n$, the element $T_{i}(x)=\left(x_{1}, \ldots, x_{i-1}, x_{i}+h_{i}^{x}, x_{i+1}, \ldots, x_{n}\right) \in P E(\mathfrak{G})$ also belongs to $P D(\mathfrak{G})$. This fact allows us to find all solutions of these Diophantine equations and also to establish some of their properties.

The mappings $T_{i}$ are involutions of the primary ellipsoid. In the group of all permutations of the primary ellipsoid, consider the subgroup $W P E(\mathfrak{G})$ generated by the mappings $T_{i}$.

Theorem 3.1. The group WPE(G) is isomorphic to the Weyl group of the Lie algebra $\mathfrak{G}$.

Proof. Follows from Coxeter relations.

Corollary 3.1. The subset $P D(\mathfrak{G})$ of the primary ellipsoid is invariant under the action of the group $W P E(\mathfrak{G})$ and it splits into orbits. The set of the orbits is one-to-one with the subset of the set of all integral solutions with all nonnegative components of the equation of the secondary ellipsoid. For any such a sollution $h$, the vector $x_{h}=A^{-1}(\tilde{1}-h)$, is the unique minimal vector of the respective orbit under the partial ordering $\leq$.

We parametrize the set of the orbits by their minimal elements. For any such vector $a=x_{h}$, denote by $P D_{a}(\mathfrak{G})$ the respective orbit. For example, the number of orbits for the case of simple Lie algebra of class $E_{8}$ is equal to 157. For the simple Lie algebras of small rank $\left(A_{2}, A_{3}, A_{4}, B_{2}, G_{2}\right)$ there is only one orbit. There is only one integral solution $h$ in the secondary ellipsoid with all nonnegative components that has all its components positive, and it is equal to $\tilde{1}$. All other such solutions have at least one component equal to 0 . For any such $h$ consider all those values of index $i_{1}<i_{2}<\ldots<i_{k}$ for which the corresponding component is equal to 0 . In the Weyl group $W$, let $W_{h}$ be the subgroup generated by the elementary reflections $e_{i_{1}}, e_{i_{2}}, \ldots, e_{i_{k}}$.

Corollary 3.2. The number of elements in the respective orbit is equal to the number $\operatorname{card}(W) / \operatorname{card}\left(W_{h}\right)$.

There is only one orbit with the number of elements equal to the order of the Weyl group. This orbit contains the origin 0 and all the vectors $m_{\alpha} \alpha$ for any positive root $\alpha$, as well as the element $2 \delta$ together with all the elements $2 \delta-m_{\alpha} \alpha$, where $m_{\alpha}$ is the grade of the positive root $\alpha$. We shall call this orbit the main orbit and denote it by $P D_{0}(\mathfrak{G})$. The corresponding subset of the secondary ellipsoid will be denoted by $S D_{0}(\mathfrak{G})$. The vectors from $P D_{0}(\mathfrak{G})$ do not have negative components, and the vectors of $S D_{0}(\mathfrak{G})$ do not have zero components.

\section{Primary and Secondary Geometric Realizations of the Weyl Group}

Denote by $W(\mathfrak{G})$ the Weyl group of the complex semisimple Lie algebra $\mathfrak{G}$. In this section we are concerned with geometric realizations of the Weyl group related to the primary and secondary ellipsoids. We first realize it in a matrix form. For any simple root $e_{i}$, the matrix corresponding to the reflexion $s_{i}$ generated by $e_{i}$ is 
determined as follows: take the i-th line of the matrix $I-A$ and replace by it the $\mathrm{i}$-th line in the matrix $I$. Denote the matrix thus obtained by $M_{i}$. This matrix is the matrix of the simple reflection $s_{i}$. The matrix group generated by the matrices $M_{1}, M_{2}, \ldots, M_{n}$ is a matrix realization of the Weyl group $W(\mathfrak{G})$. This can be shown by checking the Coxeter relations. We denote this matrix group by $M W(\mathfrak{G})$.

Assign to any element $w$ of the matrix Weyl group $M W(\mathfrak{G})$ the vector

$$
P_{w}=\delta-w \delta \text {. }
$$

Proposition 4.1. For any $w \in M W(\mathfrak{G})$, the vector $P_{w}=\delta-w \delta$ belongs to the primary ellipsoid $P E(\mathfrak{G})$.

Proof. The proof follows from a straightforward calculation.

Proposition 4.2. The mapping $w \rightarrow P_{w}$ from $M W(\mathfrak{G})$ to $P E(\mathfrak{G})$ is injective.

Proof. The proof follows from the fact that the Weyl group acts simply transitively on the set of all Weyl chambers.

Proposition 4.3. The image of a reflection $s_{\alpha}$ by the mapping $P$ is the vector $m_{\alpha} \alpha$, where $m_{\alpha}$ is the grade of the positive root $\alpha$.

Proof.

$$
\begin{aligned}
P_{s_{\alpha}} & =\delta-s_{\alpha} \delta=\frac{1}{2}\left(\sum_{\beta>0} \beta-\sum_{\beta>0} s_{\alpha} \beta\right)=\frac{1}{2}\left(\sum_{\beta>0} \beta-\sum_{\beta>0}\left(\beta-\frac{2(\beta, \alpha)}{(\alpha, \alpha)} \alpha\right)\right) \\
& =\frac{1}{2} \sum_{\beta>0} \frac{2(\beta, \alpha)}{(\alpha, \alpha)} \alpha=\frac{2(\delta, \alpha)}{(\alpha, \alpha)} \alpha=m_{\alpha} \alpha .
\end{aligned}
$$

Corollary 4.1. The image of a simple reflection $s_{i}$ by the mapping $P$ is the basis vector $e_{i}$.

Now, consider the image of the product $w=w_{1} w_{2}$ of two elements of the Weyl group $M W(\mathfrak{G})$ under the mapping $w \rightarrow P_{w}$.

$$
P_{w_{1} w_{2}}=\delta-\left(w_{1} w_{2}\right) \delta=\delta-w_{1} \delta+w_{1}\left(\delta-w_{2} \delta\right)=P_{w_{1}}+w_{1} P_{w_{2}}
$$

From this equality we also get the formula for the image of the inverse element:

$$
P_{w^{-1}}=-w^{-1} P_{w} .
$$

Corollary 4.2. The image of the Weyl group $M W(\mathfrak{G})$ by the mapping $P$ is the main orbit $P D_{0}(\mathfrak{G})$.

Motivated by formulas 29 and 30, define the group operation denoted by $*$ on the set $P D_{0}(\mathfrak{G})$ as follows. By Proposition 4.2, for any $a \in P D_{0}(\mathfrak{G})$ there exists a unique $w_{a} \in M D(\mathfrak{G})$ such that $P_{w_{a}}=a$. For any $a, b \in P D_{0}(\mathfrak{G})$ set

$$
a * b=a+w_{a} b
$$

and

$$
a^{-1}=-w_{a}^{-1} a
$$

So, we shall define $P W(\mathfrak{G})$ to be the set $P D_{0}(\mathfrak{G})$ with the transfered operation $*$, and call it the primary realization of the Weyl group. In this realization of the Weyl group the identity element is the origin $0=(0, \ldots, 0)$. Formulas 29 and 30 imply that $P W(\mathfrak{G})$ is a group isomorphic to the Weyl group $W(\mathfrak{G})$. This realization has some interesting features.

Let $a=e_{i}=(0, \ldots, 1, \ldots, 0)$ and $b=\left(b_{1}, \ldots, b_{i}, \ldots, b_{n}\right)$ an arbitrary element of $P W(\mathfrak{G})$. Then

$$
e_{i} * b=\left(b_{1}, \ldots, b_{i}+h_{i}^{b}, \ldots, b_{n}\right)=T_{i}(b),
$$

where $h_{i}^{b}$ is the $i-t h$ component of the vector $h^{b}=\tilde{1}-A b$. This property can be generalized to the case of $a_{\alpha} \in P W(\mathfrak{G})$ being equal to a multiple of a positive root $\alpha, a_{\alpha}=m_{\alpha} \alpha$, where $m_{\alpha}$ is the grade of the positive root $\alpha$ defined by Formula 8. In this case we have that

$$
a_{\alpha} * b=\left(m_{\alpha} \alpha\right) * b=\left(m_{w_{b}^{-1} \alpha}\right) \alpha+b .
$$

One more property is about multiplication on the left by the element $2 \delta$. For any element $a \in P W(\mathfrak{G})$,

$$
(2 \delta) * a=(2 \delta)-a \text {. }
$$


In particular,

$$
(2 \delta) *(2 \delta)=0 .
$$

If $e_{i}$ and $e_{j}$ are orthogonal simple roots, which means that $a_{i j}=0$, then

$$
e_{i} * e_{j}=e_{i}+e_{j} \text {. }
$$

And even more, if positive roots $\alpha$ and $\beta$ are orthogonal, then

$$
\left(m_{\alpha} \alpha\right) *\left(m_{\beta} \beta\right)=m_{\alpha} \alpha+m_{\beta} \beta .
$$

If positive roots $\alpha$ and $\beta$ are not orthogonal, then

$$
\left(m_{\alpha} \alpha\right) *\left(m_{\beta} \beta\right)=\left(m_{w_{\beta} \alpha}\right) \alpha+m_{\beta} \beta .
$$

Now we assign to any element $w$ of the Weyl group $M W(\mathfrak{G})$ the vector

$$
S^{w}=A w \delta .
$$

Proposition 4.4. For any $w \in M W(\mathfrak{G})$, the vector $S^{w}=A w \delta$ belongs to the secondary ellipsoid $S E(\mathfrak{G})$. Proof. The proof follows from a straightforward calculation.

Proposition 4.5. The mapping $w \rightarrow S^{w}$ from $M W(\mathfrak{G})$ to $S E(\mathfrak{G})$ is injective and maps $M W(\mathfrak{G})$ into $S D_{0}(\mathfrak{G})$.

Proof. The mapping $w \rightarrow S^{w}$ from $M W(\mathfrak{G})$ to $S E(\mathfrak{G})$ can be represented as a composition of the mappings $w \rightarrow \delta-w \delta$ from $M W(\mathfrak{G})$ to $P D(\mathfrak{G})$ and the mapping $x \rightarrow \tilde{1}-A x$ (which maps $P D_{0}(\mathfrak{G})$ into $\left.S D_{0}(\mathfrak{G})\right)$, because

$$
A w \delta=\tilde{1}-A(\delta-w \delta)
$$

Theorem 4.1. The mapping $w \rightarrow S^{w}=A w \delta$ from $M W(\mathfrak{G})$ to $S D_{0}(\mathfrak{G})$ is a bijection.

Proof. This is obvious for those cases $\left(G_{2}, F_{4}, E_{8}\right)$ where det $A=1$. In all the other cases, the proof follows from a case-by-case consideration.

The mapping $w \rightarrow S^{w}=A w \delta$, being a bijection, transfers the group structure from the Weyl group $M W(\mathfrak{G})$ to the set $S D_{0}(\mathfrak{G})$ thus producing the secondary geometric realization of the Weyl group $W(\mathfrak{G})$, which we shall denote by $S W(\mathfrak{G})$. In this realization of the Weyl group, the identity element is $\tilde{1}$, and the element of maximal length is $-\tilde{1}$.

\section{Orderings of the Weyl Group}

The realization $P W(\mathfrak{G})$ of the Weyl group on the primary ellipsoid provides a partial ordering of this group that is inherited from the natural partial ordering of the linear space $\mathbb{R}^{n}$. A vector $x=\left(x_{1}, \ldots, x_{n}\right)$ is less or equal in this ordering than $y=\left(y_{1}, \ldots, y_{n}\right)$ if and only if for all $i$ we have $x_{i} \leq y_{i}$. We denote this ordering by $x \leq y$ and call it the primary ordering of the group $P W(\mathfrak{G})$. In this ordering of the group $P W(\mathfrak{G})$, there is a unique minimal element $0=(0, \ldots, 0)$, and a unique maximal element $2 \delta$. Formula 33 implies that for any $b \in P W(\mathfrak{G})$ and for any $i$ the elements $b$ and $e_{i} * b$ are comparable under the primary ordering; if $0 \leq h_{i}^{b}$, then $b \leq e_{i} * b$, and the inequality reverses otherwise. This statement can be generalized, by using Formula 39, to the case when we take any positive root $\alpha$ and its grade $m_{\alpha}$, and consider the element $a_{\alpha}=m_{\alpha} \alpha$ of the group $P W(\mathfrak{G})$ and an arbitrary element $b \in P W(\mathfrak{G})$. The product $a_{\alpha} * b$ and the element $b$ are also comparable.

There is another very important for different applications ordering for any Weyl group, which is called Chevalley-Bruhat ordering, see [4]-[9], and which we denote by $\prec$. To define the Chevalley-Bruhat ordering, we first need to define the length of an element $w$ of a Weyl group. The element $w$ can be written as a product of elementary reflections $s_{i}$. This can be done in several different ways. The minimal number of factors in such a representation of $w$ is called the length of $w$ (notation $l(w)$ ). An expression of $w$ as a product of elementary reflections with the number of factors equal to the length of $w$ is called a reduced expression. The ellipsoid geometric realizations of the Weyl group provide a way to find the length of any element $w$ of any Weyl group and also the family of all its reduced expressions. Denote by $Z_{t}$ the family of all reflections in $W(\mathfrak{G})$ with respect to positive roots. For $a, b \in W(\mathfrak{G})$ and $t \in Z$, write $a \rightarrow b$ if $b=t a$ and $l(a)<l(b)$. In turn, write 
$a \rightarrow b$ if $\stackrel{t}{\rightarrow} b$ for some $t \in Z$. Extend this relation to a partial ordering on $W(\mathfrak{G})$ by defining $a \prec b$ to mean

$a=a_{0} \rightarrow a_{1} \rightarrow \ldots \rightarrow a_{n}=b$ for some $a_{1}, a_{2}, \ldots, a_{n-1}$.

In the realization of the Weyl group on the pimary ellipsoid, we have that $a \prec b=t * a$ with $t$ being a reflection with respect to a positive root $\alpha$, so that $t=m_{\alpha} \alpha$, we have that,

$$
b=a+k_{a}^{\alpha} \alpha,
$$

with

$$
k_{a}^{\alpha}=\frac{2<\alpha, \delta-a\rangle}{\langle\alpha, \alpha>} .
$$

By definition, $a=\delta-w \delta$ for some $w \in M W(\mathfrak{G})$, therefore,

$$
k_{a}^{\alpha}=\frac{2<\alpha, w \delta>}{<\alpha, \alpha>}=m_{w^{-1} \alpha} .
$$

This implies that $a \stackrel{t}{\rightarrow} b$ if and only if $w^{-1} \alpha$ is a positive root. Thus $a \stackrel{t}{\rightarrow} b$ implies that $a \leq b$, and so $a \prec b$ implies that $a \leq b$.

In this ordering, 0 is the unique minimal element too, and $2 \delta$ is the unique maximal.

As a matter of fact, the primary ordering is in some cases strictly stronger than the Chevalley-Bruhat ordering on the Weyl group, as one can see, for example, from the case of $A_{3}$. In this case, the Weyl group is isomorphic to the symmetric group $S_{4}$, and the graph of the Chevalley-Bruhat ordering for this group is available in Fig. 2.4 of [4]. When compared to the primary ordering, it can be seen that there are two cases of discrepancy bitween the two orderings for this Weyl group. In the case of the Chevalley-Bruhat ordering, the elements (1 43 2) and (4 $\left.12 \begin{array}{lll}2 & 3\end{array}\right)$ of $S_{4}$ are not comparable, as can be seen from Fig. 2.4 of [4], but their respective counterparts in the primary ellipsoid geometric realization are the vectors $(0,2,2)$ and $(1,2,3)$, which are comparable in the primary ordering. The same holds true for the elements (3 $\left.2 \begin{array}{lll}2 & 1\end{array}\right)$ and $\left(\begin{array}{llll}2 & 3 & 4 & 1\end{array}\right)$ of $S_{4}$, which have the vectors $(2,2,0)$ and $(3,2,1)$ as their respective counterparts. In all the other cases, the two orderings agree for $A_{3}$.

Observe that in these two cases of $a \leq b$ but not $a \prec b$ we have that $b-a=(1,0,1)$, which is not a multiple of a positive root.

To Chevalley-Bruhat order a Weyl group $W$ by using the primary realization take the following steps: 1) Realize $W$ primarily by assigning $P(w)=\delta-w \delta$ to any $w$; 2) Order the primary realization primarily by inserting a link between any two directly adjacent elements $a \leq b$; 3) Delete all those links with $a \leq b$ for which $b-a$ is not a multiple of a positive root. The remaining links provide the Chevalley-Bruhat ordering of the Weyl group $W$.

The secondary realization of a Weyl group provides an efficient way to obtain all reduced expressions for any ellement $w$ of the Weyl group. A reduced expression of $w$ is a shortest possible expression of it as a product of simple reflections $s_{i}$. Finding all reduced expressions of any element of a Weyl group boils down to finding the first element in any such expression, because if $s$ is known to be the first element of a reduced expresion for $w$, to find the second element of this reduced expression is equivalent to finding the first element of the product sw, and so on.

Theorem 5.1. Given an element $w$ of the Weyl group $M W(\mathfrak{G})$, consider its image $S(w)=A w \delta$ in $S W(\mathfrak{G})$. The vector $S(w)$ being an $n$-tuple of positive and negative integers $\left(b_{1}, b_{2}, \ldots, b_{n}\right)$, let $i_{1}<i_{2}<. .<i_{m}$ be the values of index $i$ for which $b_{i}$ is negative. Then $s_{i_{1}}, s_{i_{2}}, \ldots, s_{i_{m}}$ are the only simple reflections that can be the first elements of a reduced expression of $w$.

Proof. This follows directly from the definition of the secondary realization of the Weyl group.

This theorem provides an alternative way to build the Chevalley-Bruhat ordering of a Weyl group, because, as is well known for any Coxeter group (see for example [8]), knowing reduced expressions leads to ChevalleyBruhat ordering through subexpressions.

\section{References}

[1] Jacobson, N. (1962) Lie Algebras. Interscience, New York.

[2] Knapp, A.W. (1996) Lie Groups Beyond an Introduction. Birkhauser, Boston, Basel, Berlin. 
http://dx.doi.org/10.1007/978-1-4757-2453-0

[3] Bourbaki, N. (1968) Elements de Mathematiques, Groupes et Algebres de Lie: Chapitres 4,5, et 6. Actualites Scientifiques et Industrielles 1337, Hermann, Paris.

[4] Bjorner, A. and Brenti, F. (2005) Combinatorics of Coxeter Groups. Springer-Verlag, New York.

[5] Deodhar, V.V. (1977) Some characterizations of Bruhat ordering on a Coxeter Group and Determination of the Relative Mobius Function. Inventiones Mathematicae, 39, 187-198. http://dx.doi.org/10.1007/BF01390109

[6] Deodhar, V.V. (1985) On Some Geometric Aspects of Bruhat Ordering, I. A Finer Decomposition of Bruhat Cells. Inventiones Mathematicae, 79, 499-511. http://dx.doi.org/10.1007/BF01388520

[7] Humphreys, J.E. Representations of Semisimple Lie algebras in the BGG Cathegory O. GSM, 94, AMS.

[8] Humphreys, J.E. (1990) Reflection Groups and Coxeter Groups. Cambridge University Press, Cambridge. http://dx.doi.org/10.1017/CBO9780511623646

[9] Kazhdan, D. and Lusztig, G. (1979) Representations of Coxeter Groups and Hecke Algebras. Inventiones Mathematicae, 53, 165-184. http://dx.doi.org/10.1007/BF01390031

\section{Submit or recommend next manuscript to SCIRP and we will provide best service for you:}

Accepting pre-submission inquiries through Email, Facebook, LinkedIn, Twitter, etc.

A wide selection of journals (inclusive of 9 subjects, more than 200 journals)

Providing 24-hour high-quality service

User-friendly online submission system

Fair and swift peer-review system

Efficient typesetting and proofreading procedure

Display of the result of downloads and visits, as well as the number of cited articles

Maximum dissemination of your research work

Submit your manuscript at: http://papersubmission.scirp.org/ 